\title{
Clinical and molecular epidemiology of beta-hemolytic streptococcal infections in India
}

\author{
Purva Mathur, Nidhi Bhardwaj, Kushal Mathur, Bijayini Behera, Gunjan Gupta, Arti Kapil, Sarman Singh, \\ Mahesh C. Misra
}

All India Institute of Medical Sciences, New Delhi, India

\begin{abstract}
Introduction: Beta-hemolytic streptococci $(\beta \mathrm{HS})$ cause a diverse array of human infections. Despite the high number of cases of streptococcal carriers and diseases, studies discerning the molecular epidemiology of $\beta \mathrm{HS}$ in India are limited. This study reports the molecular and clinical epidemiology of beta-hemolytic streptococcal infections from two geographically distinct regions of India.

Methodology: A total of 186 isolates of $\beta \mathrm{HS}$ from north and south India were included. The isolates were identified to species level and subjected to antimicrobial susceptibility testing. Polymerase chain reaction (PCR) was done to detect exotoxin genes, and emm types of group A streptococci (GAS) strains were ascertained by sequencing.

Results: GAS was the most common isolate (71.5\%), followed by group G streptococci (GGS) (21\%). A large proportion of GAS produced speB (97\%), smeZ (89\%), speF (91\%), and speG (84\%). SmeZ was produced by $21 \%$ and $50 \%$ of GGS and GGS, respectively. A total of 45 different emm types/subtypes were seen in GAS, with emm 11 being the most common. Resistance to tetracycline (73\%) and erythromycin (34.5\%) was commonly seen in GAS.

Conclusions: A high diversity of emm types was seen in Indian GAS isolates with high macrolide and tetracycline resistance. SpeA was less commonly seen in Indian GAS isolates. There was no association between disease severity and exotoxin gene production.
\end{abstract}

Key words: beta-hemolytic streptococci; group A streptococcus; Spe; emm types; antimicrobial resistance

J Infect Dev Ctries 2014; 8(3):297-303. doi:10.3855/jidc.3216

(Received 12 December 2012 - Accepted 10 September 2013)

Copyright (C) 2014 Mathur et al. This is an open-access article distributed under the Creative Commons Attribution License, which permits unrestricted use, distribution, and reproduction in any medium, provided the original work is properly cited.

\section{Introduction}

Beta-hemolytic streptococci ( $\beta \mathrm{HS})$ are known to cause a diverse set of clinical presentations. Group A streptococci (GAS) constitute the most important and prevalent members of this group, causing clinical syndromes ranging from self-limited pharyngitis and impetigo to potentially life-threatening toxic shock syndrome [1]. Invasive GAS infections have increased worldwide in the recent past, despite the organism remaining sensitive to penicillin and other commonly used beta-lactam antibiotics. Group B streptococci (GBS) are a leading cause of infections in neonates and pregnant women and also cause invasive diseases in children and non-pregnant adults. Group C (GCS) and group G (GGS) streptococci (together known as GCGS) are usually commensals of the pharynx, skin, gastrointestinal tract, and female genital tract. Large colony phenotypes of GCGS are usually associated with human infections and are classified in the same subspecies, Streptococcus dysgalactiae subsp. Equisimilis subsp. Nov (SDSE) [2], whereas the small colony forming species are placed in the Streptococcus anginosus group (SAG, formerly known as Streptococcus milleri) and are less common causes of invasive infections [3]. The SDSE group is gaining importance because the clinical spectrum of diseases caused by them closely resembles $S$. pyogenes infections.

Among the many factors involved in the pathogenesis of GAS, the exotoxins (superantigens) and $\mathrm{M}$ proteins are the most important. The toxins secreted by $S$. pyogenes are antigenically distinct extracellular products and include speA, speB, speC, speG, speH, speI, speK, speJ, speF, speL, smeZ, and ssa [4]. These toxins are associated with various immunological and cytotoxic effects. Most of the strains associated with invasive streptococcal infections are reported to carry speA.

The emm gene of GAS encodes its major virulence factor, the $\mathrm{M}$ protein. It shows marked variability in its hypervariable region, accounting for its diversity, which forms the basis for emm typing [1,5]. Sequence 
analysis of the emm gene is an important surveillance tool for understanding the dynamics of local transmission of GAS. The distribution of emm types shows tremendous geographic variation. Thus, it is essential to know the emm-type distribution of different patient populations in various parts of the same country. This would enable understanding of the local epidemiology and the formulation of indigenous vaccines. In India, compared to the disease burden [6], relatively few studies have been conducted on the overall profile of beta-hemolytic streptococcal infections, the distribution of emm types, and the presence of exotoxins. Since India has tremendous ethnic and geographic diversity, studies encompassing different regions of the country would give more meaningful epidemiological information.

We therefore conducted this study to ascertain the clinical and molecular epidemiology of streptococcal infections from two geographically distinct areas (north and south) of India. We also wanted to ascertain if there is any correlation between exotoxin profile and the relatively low reported prevalence of invasive streptococcal infections in India, which has a high number of cases and carriers of streptococcal pharyngitis.

\section{Methodology}

The study was conducted at the Microbiology Laboratory of the level 1 Jai Prakash Narayan Apex (JPNA) Trauma Center - which contains 165 beds - of the All India Institute of Medical Sciences (AIIMS), New Delhi, a 2,000-bed tertiary care hospital in India. The $\beta H S$ included in this study were recovered from clinical samples from different hospitals and communities of India, as detailed below.

\section{$\beta H S$ recovered from trauma patients in north India}

All the $\beta$ HS $(n=85)$ recovered over a period of three years (between April 2009 and March 2012) from various clinical samples of patients admitted to the Trauma Centre, AIIMS, and from those presenting to its follow-up outpatient department (OPD), were included in the study. A detailed clinical history was recorded for all these patients and post-treatment follow-up was done. A throat swab was taken from all the patients whose cultures yielded $\beta \mathrm{HS}$ from any sample other than throat to look for colonization. A repeat sample was also taken from the site of primary isolation after five days of appropriate antimicrobial treatment to look for a microbiological cure. $\beta H S$ recovered from patients with pharyngitis/tonsillitis

A total of 14 isolates of $\beta$ HS were obtained from patients presenting with symptoms of acute pharyngitis/tonsillitis to an outdoor clinic situated in a heavily populated area of old Delhi between April 2009 and March 2012.

\section{$\beta H S$ recovered from a south Indian hospital}

All $\beta$ HS $(n=23)$ recovered from various clinical samples between June 2010 and March 2012 at the microbiology laboratory of Yashoda hospital, Secunderabad (situated in southeast India, with 500 beds) were included in the study. A detailed history of all patients was taken and clinic-microbiological follow-up was done similar to the trauma center patients.

\section{$\beta H S$ recovered from AIIMS hospital, north India}

All $\beta$ HS isolated at the microbiology laboratory of the AIIMS hospital (bed strength, 2,000) from pus samples between 2005 and $2009(\mathrm{n}=64)$ were preserved for further studies. These isolates were also included in the present study. However, detailed histories of these patients was not available.

The study was approved by the institute's ethical committee.

All the isolates of $\beta$ HS were identified by standard microbiological methods [7]. The confirmation of identity was done using the Vitek 2 identification cards (bioMérieux, Marcy l'Etoile, France). Grouping of the streptococci was performed using an agglutination test (HiMedia Labs, Mumbai, India) according to the manufacturer's instructions. All the strains were stocked in stocking beads (Microbank, Pro-Lab Diagnostics, Richmond Hill, Canada) at $70^{\circ} \mathrm{C}$ until further analysis.

\section{Antimicrobial susceptibility testing}

The antimicrobial susceptibility testing of streptococci was performed using the disk diffusion method on Mueller Hinton agar with 5\% sheep's blood according to the recommendations of the CLSI $[8,9]$. The following antibiotics were tested: penicillin G, ampicillin vancomycin, erythromycin, clindamycin, cefotaxime, ceftriaxone, linezolid, teichoplanin, ciprofloxacin, levofloxacin, tetracycline, and chloramphenicol. S. pneumoniae ATCC 49619 was used as a control. The minimum inhibitory concentration (MIC) was also determined by the E-test for all the above antimicrobials; the test was performed according to manufacturer's 
recommendations (bioMérieux, Marcy l'Etoile, France).

\section{Detection of virulence genes}

Exotoxin genes, including spe A, speB, speC, speF, smeZ, ssa, speG, speH, speJ, speL, speM, and speI were detected by PCR using published primers $[10,11]$. Amplification of all the genes was performed with an initial five-minute denaturation at $94^{\circ} \mathrm{C}$, followed by 30 cycles of denaturation at $94^{\circ} \mathrm{C}$ for 30 seconds, 30 seconds of annealing at temperatures standardized in the laboratory for each gene, and 60 seconds of extension at $72^{\circ} \mathrm{C}$, with a final extension step at $72^{\circ} \mathrm{C}$ for seven minutes.

\section{Emm typing of S. pyogenes}

The emm types of isolates of $S$. pyogenes were determined by sequencing the variable 5' end of emm gene after amplification by PCR. For this, the DNA preparation of GAS strains was performed as described by the United States Centers for Disease Control and Prevention (CDC). The amplification of the emm gene and sequencing was performed as per the published protocol [12]. Amplification of the emm genes was performed by the all $\mathrm{M}$ primer having the following sequence:

Forward primer 5'-GGG GGG GGA TCC ATA
AGG AGC ATA AAA ATG GCT-3'; reverse primer: 5'- GGG GGG GAA TTC AGC TTA GTT TTC TTC TTT GCG-3'.

The emm gene sequence was subjected to homology search by blast search analysis (http://www.ncbi.nlm.nih.gov/BLAST/Blast.cgi). A comparison of the nucleotide homology for the first 200 bases of the hypervariable region was conducted. Strains that showed $\geq 95 \%$ homology with the reference strain were designated the particular parental emm type. Types and subtypes (based on variation in the type-specific region of the emm gene) were designated according to the information available on the CDC website (http://www.cdc.gov/ncidod/biotech/strep/doc.htm).

\section{Results}

During the study period, a total of 186 isolates of $\beta \mathrm{HS}$ were obtained from various clinical samples and places of study as detailed in the methodology. The distribution of various $\beta \mathrm{HS}$ from the different places of study and samples is shown in Table 1. Repeated isolation of the same $\beta \mathrm{HS}$ from the same site was considered to be a single isolate. The sample-wise distribution of various groups of $\beta \mathrm{HS}$ is shown in Table 2. Pus was the most common source of $\beta \mathrm{HS}$ in our study (142; 76\%).

Table 1: Distribution of samples yielding beta-hemolytic streptococci from various hospitals

\begin{tabular}{|c|c|c|c|c|c|c|c|c|c|c|}
\hline Hospital/clinic & $\begin{array}{c}\text { Pus / } \\
\text { wound } \\
\text { swab }\end{array}$ & Throat & Blood & $\begin{array}{l}\text { Tracheal } \\
\text { aspirate }\end{array}$ & $\begin{array}{c}\text { Bronchial } \\
\text { wash }\end{array}$ & Sputum & $\begin{array}{c}\text { Graft } \\
\text { sample }\end{array}$ & Tissue & $\begin{array}{l}\text { Blister } \\
\text { fluid }\end{array}$ & $\begin{array}{r}\text { Total } \\
\text { N }(\%)\end{array}$ \\
\hline $\begin{array}{l}\text { Trauma Centre, AIIMS } \\
\text { Throat swabs from }\end{array}$ & 68 & 8 & 4 & 3 & & & 1 & 1 & & $85(46)$ \\
\hline $\begin{array}{l}\text { patients with pharyngitis } \\
\text { (outdoor clinic in old } \\
\text { Delhi) }\end{array}$ & & 14 & & & & & & & & $14(7.5)$ \\
\hline $\begin{array}{l}\text { Yashoda Hospital, } \\
\text { Secunderabad (admitted } \\
\text { and outdoor patients) } \\
\text { AIIMS hospital }\end{array}$ & 10 & 4 & 3 & & 1 & 3 & & 1 & 1 & $23(12)$ \\
\hline $\begin{array}{c}\text { (preserved strains } \\
\text { obtained from pus } \\
\text { samples over five years) }\end{array}$ & 64 & & & & & & & & & $64(34)$ \\
\hline $\begin{array}{l}\text { Total } \\
\mathrm{n}(\%)\end{array}$ & $\begin{array}{c}142 \\
(76 \%)\end{array}$ & $\begin{array}{c}26 \\
(14)\end{array}$ & $\begin{array}{c}7 \\
(4)\end{array}$ & $\begin{array}{c}3 \\
(2)\end{array}$ & $\begin{array}{c}1 \\
(0.5)\end{array}$ & $\begin{array}{c}3 \\
(2)\end{array}$ & $\begin{array}{c}1 \\
(0.5)\end{array}$ & $\begin{array}{c}2 \\
(1)\end{array}$ & $\begin{array}{c}1 \\
(0.5)\end{array}$ & 186 \\
\hline
\end{tabular}

Table 2: Distribution of various groups of beta-hemolytic streptococci from different samples

\begin{tabular}{|c|c|c|c|c|c|c|c|c|c|c|}
\hline $\begin{array}{l}\text { Streptococcal } \\
\text { group }\end{array}$ & Pus/wound & Throat & Blood & Tracheal & $\begin{array}{l}\text { Bronchial } \\
\text { wash }\end{array}$ & Sputum & Graft & $\begin{array}{l}\text { Blister } \\
\text { fluid }\end{array}$ & Tissue & Total n (\%) \\
\hline GAS & 108 & 18 & 4 & & & & 1 & 1 & 1 & $133(71.5)$ \\
\hline GGS & 24 & 7 & 1 & 3 & 1 & 2 & & & 1 & $39(21)$ \\
\hline GCS & 5 & & & & & 1 & & & & $6(3)$ \\
\hline GFS & & 1 & 1 & & & & & & & $2(1)$ \\
\hline GBS & 5 & & 1 & & & & & & & $6(3)$ \\
\hline Total & 142 & 26 & 7 & 3 & 1 & 3 & 1 & 1 & 2 & 186 \\
\hline
\end{tabular}




\section{Clinical profile of patients}

Of the 186 isolates of $\beta \mathrm{HS}$ included in the study, a total of $64(34 \%)$ represented preserved strains from pus samples over a period of five years (the detailed history of these patients was unavailable), and 29 $(15.5 \%)$ isolates were recovered from throat swabs or sputum of patients suffering from acute pharyngitis. The remaining $93 \beta \mathrm{HS}$ were recovered from various invasive infections (77 of these were recovered from various samples of trauma patients and 16 were from various clinical samples of patients admitted to Yashoda hospital). Of the 77 isolates from trauma patients, $70 \quad(91 \%)$ were recovered from pus/tissue/graft samples. The primary sites of trauma from which $\beta$ HS were recovered in these patients were lower limb $(41 ; 59 \%)$, spine $(9 ; 13 \%)$, head/scalp (7; $10 \%$ ), abdomen $(4 ; 6 \%)$, upper limbs $(3 ; 4 \%)$, mandible and chest (2;3\% each), and vascular injury and pelvic trauma $(1 ; 1 \%$ each).

Of these 93 patients, a total of five $(5 \%)$ had a fatal outcome. Septicemia was the cause of death in all these patients. One of these patients had severe head injury and septicemia secondary to Ventilator associated pneumonia (VAP). GAS was isolated from his pus sample. Three patients had GGS isolated from their pus/abscess material, and all of them died due to septic shock. In one of them, GGS was isolated initially from pus aspirated from a para-aortic abscess and later from blood. One patient had septicemia due to GFS following a decompressive craniectomy.

\section{Throat colonization as probable source of infection}

Throat swabs were taken from all patients who had positive $\beta H S$ cultures from specimens other than throat. Three patients had the same $\beta$ HS (GAS/GGS) from throat swabs. The strains of GAS from pus and throat (two patients) were identical in susceptibility, exotoxin profile, and $\mathrm{emm}$ types and were therefore considered to be the same strain. The strains of GGS from tracheal aspirate and throat (one patient) were also identical in susceptibility and exotoxin profile.

\section{Beta-hemolytic streptococcal groups and species}

GAS was the most common $\beta \mathrm{HS}$ (71.5\%), followed by group G (21\%) (Table 2). All the 133 GAS were identified as $S$. pyogenes. Of the 39 GGS, $26(67 \%)$ were identified as $S$. dysgalactiae, six (15\%) as $S$. anginosus, four $(10 \%)$ as $S$. dysgalactiae subsp.Equisimilis, and one (2.5\%) each as $S$. porcinus, $S$. alactolyticus, and $S$. mitis. Of the six GCS, five (83\%) were $S$. dysgalactiae, and one was $S$. dysgalactiae subsp.Equisimilis. One isolate each of
GFS was identified as $S$. anginosus and $S$. constellatus. All the six isolates of GBS were identified as $S$. agalactiae.

\section{Antimicrobial susceptibility of $\beta H S$}

All isolates were sensitive to penicillin, ampicillin, vancomycin, cefotaxime, ceftriaxone, clindamycin, linezolid, teichoplanin, and levofloxacin. The MIC of penicillin ranged from 0.003 to $0.047 \mu \mathrm{g} / \mathrm{mL}$ (median MIC $0.008 \mu \mathrm{g} / \mathrm{mL})$. A total of $97(73 \%)$ of GAS were resistant tetetracycline, $46(34.5 \%)$ to erythromycin, $18(13.5 \%)$ to chloramphenicol, and $17(13 \%)$ to ciprofloxacin. The MIC range for ciprofloxacin was 0.25 to $>32 \mu \mathrm{g} / \mathrm{mL}$ (median $0.75 \mu \mathrm{g} / \mathrm{mL}$ ). The MIC of erythromycin ranged from 0.004 to $>256 \mu \mathrm{g} / \mathrm{mL}$ (median $0.064 \mu \mathrm{g} / \mathrm{mL}$ ) and that of tetracycline ranged from 0.064 to $64 \mu \mathrm{g} / \mathrm{mL}$ (median $8 \mu \mathrm{g} / \mathrm{mL}$ ). Of the 39 strains of GGS, resistance to erythromycin, ciprofloxacin, and tetracycline was seen in two (5\%), four $(10 \%)$, and eight $(20.5 \%)$ strains, respectively. All the strains of GCS and GFS were resistant to tetracycline, and were sensitive to other agents. Among the GBS, three isolates (50\%) were resistant to tetracycline, and one each to erythromycin, ciprofloxacin, and chloramphenicol.

\section{Treatment and response}

For the infections caused by $\beta \mathrm{HS}$, the treatment consisted of debridement along with specific antimicrobials in $26(28 \%)$ of the 93 patients who had invasive infections. Antimicrobials alone were given to $67(72 \%)$ patients. Thus, all patients received antibiotics for their infections, singly or in combination. The antimicrobial regimen was guided by the antimicrobial susceptibility, prescription policy of the respective hospital/specialty, history of drug allergy, non-response to original regimen, and the clinical condition of the patient or any other concomitant infection. Thus, amoxicillin-clavulinic acid was administered to 38 (41\%) patients, clindamycin to $32(34 \%)$, cefuroxime to $29(31 \%)$, amikacin to $11(12 \%)$, cefotaxime and levofloxacin to 7 each $(7.5 \%$ each), linezolid and cefoperazone to 3 each $(3 \%$ each), vancomycin to $1(1 \%)$, and erythromycin to 1 patient. In six patients, the posttreatment samples were also positive for the same $\beta$ HS. However, all of them eventually recovered (by the third month of follow-up). 
Table 3: Exotoxin gene profile of beta-hemolytic streptococci



Table 4: Geographic and sample-wise break-up of GAS isolates with respect to exotoxin production

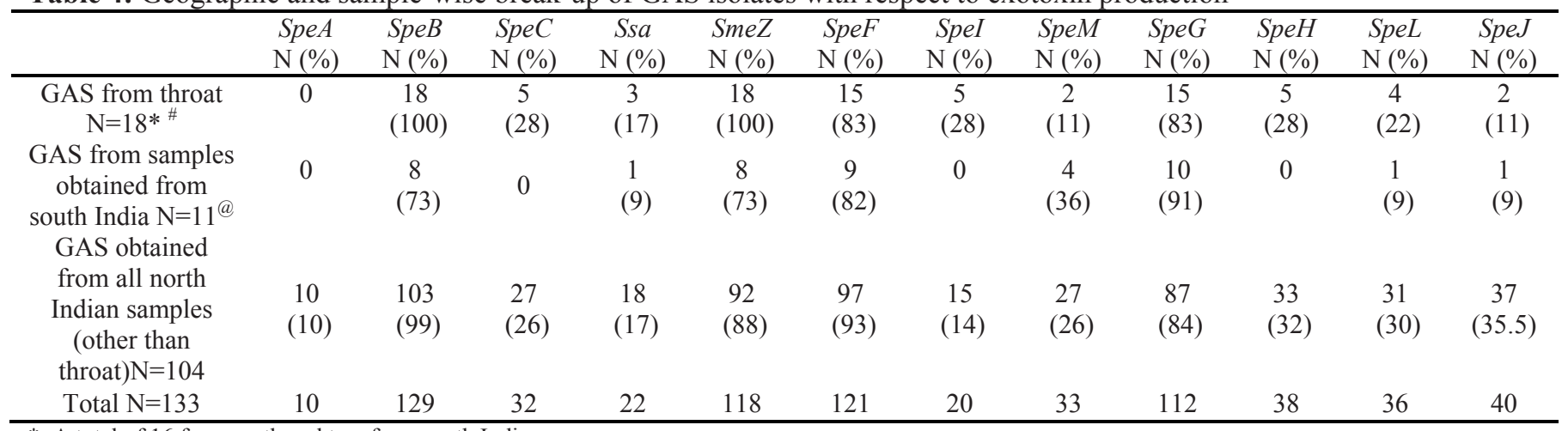

*: A total of 16 from north and two from south India

\#: Refer to Table 2

@: A total of 13 strains were obtained from south India; two were from throat swabs, which have been included with the throat isolates (see *)

Table 5: Geographic and sample-wise distribution of GAS emm types from India $(\mathrm{N}=126)^{*}$

\begin{tabular}{lll}
\hline Sample/geographic origin of GAS & Emm type & $\begin{array}{l}\text { Number of isolates } \\
N=126\end{array}$ \\
\hline South Indian isolates & & 1 each \\
Throat $(n=2)$ & 49.4 and 110 & 2 \\
Other samples $(n=10)$ & 28 & 1 each \\
& $11,25,42,77,92,101,109,109.1$ & 4 each \\
North Indian isolates & 4.5 and 12 & 1 each \\
Throat $(n=15)$ & $42,68,77,81,85,88,92$ & 13 \\
& 11 & 9 \\
Other samples $(n=99)$ & 101 & 7 \\
& 110 & 6 \\
& 92 & 4 each \\
& $25,28,112.2$ & 3 each \\
& $4.5,65,109.1$ & 2 each \\
& $25.1,57,58,71,75,77,88.3,100,112$ & 1 each \\
\hline
\end{tabular}

\footnotetext{
* Of the 133 GAS isolates, sequencing was done for 126 strains
} 


\section{Exotoxin gene profile}

All the isolates were tested for the presence of speA, speB, speC, spe $G$, speH, speI, speL, speJ, speF, speM, smeZ, and ssa by PCR. The exotoxin gene profile of the $\beta \mathrm{HS}$ is summarized in Table 3. The exotoxin gene profile of GAS from different samples and geographic origins is compared in Table 4.

Five patients in our study died, three of whom had infections due to GGS ( $S$. dysgalactiae and $S$. porcinus), and one each due to GAS and GFS ( $S$. constellatus), respectively. The strain of GFS produced speI, speM, speG, and speH. However, all the strains of GGS were negative for all the 12 exotoxins tested. The fifth patient who died had GAS from pus samples along with other complications. This strain was typed as emm 28 and was positive for speB, smeZ, and speF.

\section{Emm types of $G A S$}

Sequencing of the emm gene was performed for a total of 126 GAS isolates. Emm $11(14 ; 11 \%)$ was the most common, followed by emm $101(10 ; 8 \%)$, emm 110 and 92 (8; 6\% each), emm 4.5 (7; $5.5 \%)$, emm 28 (6; 4.7\%), emm 12 and 25 (5; 4\% each), emm 77, 109.1 and 112.2 (4; 3\% each), emm 42 and $65(3 ; 2 \%$ each), emm 25.1, 49.4, 57, 58, 68, 71, 75, 85, 88, 88.3, 100,109 and 112 (2; 1.5\% each), and emm 15, 18.12, $42.1,48,49.3,50,56,63,80,81,82.1,87,88.2,89$, $89.11,91,112.1,118,123$ (1; $0.7 \%$ each). The geographic and sample-wise distribution of emm types is shown in Table 5. The sequences have been recently submitted to NCBI's GenBank database. At the time of submission of this manuscript, one strain (emm12) had been assigned a GenBank Accession number (JQ726490).

\section{Discussion}

In this study, the $\beta H S$ strains were isolated from community and hospitalized patients from north and south India, two regions with different ethnic and socio-cultural backgrounds. To our knowledge, this is the first study discerning the clinical-molecular epidemiology of invasive and non-invasive $\beta$ HS from different regions of India. In our study, $15.5 \%$ of strains represented community isolates from patients with pharyngitis, and $12 \%$ were from a southeast Indian state that had not been represented in any such study from our country. GAS was the most common isolate in our study, although we found a high a proportion of GCGS. Similar observations had been made in a few other studies from south India [13]. The clinical presentation of GGS often mimics GAS, which needs to be considered when formulating a GAS vaccine. Since only a few laboratories identify GCGS to the species level, the actual prevalence of human SDSE and SAG infections in the Indian population may not be clear.

We found high rates of resistance in GAS to erythromycin and tetracycline, similar to reports from other parts of the world $[14,15]$. This was higher than our previously reported rates from 1996-2000 [16]. We also observed a high rate of resistance to fluoroquinoles in GAS, which may necessitate reformulating therapeutic strategies for streptococcal infections. In our study, $21 \%$ and $10 \%$ of GGS were resistant to tetracycline and ciprofloxacin, respectively. Tetracycline no longer represents an option for their empirical treatment since resistance rates up to $60 \%$ and higher have been reported [17].

The streptococcus pyrogenic exotoxins (SPEs) of GAS, especially speA, play an important role in the pathogenesis of invasive infections. A large proportion of GAS isolates in our study produced smeZ, speG, $s p e B$, and $s p e F$;relatively fewer produced speA (10\%) and speC (24\%). The speA, speC, and ssa are carried on mobile elements and are easily disseminated to other GAS. We did not find speA in any throat samples or in any samples from south India. However, $28 \%$ of strains from throat samples in our study carried speC. There was a statistically significant difference between the south and north Indian strains with respect to production of speH $(\mathrm{p}=0.03)$. The statistical association was not very strong for the difference between north and south India for $\operatorname{speC}(\mathrm{p}=$ $0.06)$ and speJ $(\mathrm{p}=0.09)$. No statistically significant difference was found for speA $(\mathrm{p}=0.5)$ and $\operatorname{speI}(\mathrm{p}=$ 0.3 ), though none of the south Indian strains produced speA, speC, or speI. Although STSS is less commonly reported in India than in Western countries, toxigenic strains in the throat may serve as reservoirs for epidemic transmission and severe invasive infection. SmeZ was produced by $21 \%$ and $50 \%$ of GGS and GCS isolates, respectively. Genes encoding speA, $s p e C$, ssa, and smeZ have been reported in SDSE strains and are nearly identical to the $S$. pyogenes gene. In our present study, the SDSE isolates recovered from fatal cases of septicemia did not carry any streptococcal superantigens. Based on our and a few other reports [18], it can be said that there is no clear-cut relationship between GCGS isolates harboring spe and disease severity, although this may be due to limited numbers of spe-positive isolates.

Our study found tremendous heterogeneity among GAS strains from north and south India, with 45 
different types/subtypes. GAS vaccines are primarily based on using multiple $M$ types to provide broad spectrum coverage. Hu et al. included 26 different $\mathrm{M}$ types in a multiple epitope formulation [19], which has been predicted to provide protection against $85 \%-90 \%$ of $\mathrm{M}$ types in the United States and Europe. In contrast to the United States and Europe, Asian countries show marked diversity of emm genotypes [20,21]. Countries such as India, Brazil, Nepal, and Australia are unlikely to benefit from the 26-valent vaccine because of this diversity [22]. Also, tailoring a vaccine for these countries will be costly and with doubtful benefits, since non-vaccine strains may colonize the vaccinated population and alter the epidemiology within a few years.

To conclude, a high prevalence of macrolide and tetracycline resistance along with marked emm-type diversity was observed in Indian GAS isolates. There was no clear association between exotoxin production and disease severity of beta-hemolytic streptococcal infections.

\section{Acknowledgements}

We acknowledge the technical help provided by Ms. Neelu, Ms. Rajrani, Ms. Sweety, MrPawan, Mr Tirlok, and Mr Vineet.

\section{References}

1. Musser JM, Shelburne SA (2009) A decade of molecular pathogenomic analysis of group A Streptococcus. J Clin Invest 119: 2455-2463.

2. Vandamme P, Pot B, Falsen E, Kersters K, Devriese LA (1996) Taxonomic study of Lancefield streptococcal groups C, G, and L (Streptococcus dysgalactiae) and proposal of $S$. dysgalactiae subsp. equisimilis subsp. nov. Int J Syst Bacteriol 46: 774-781.

3. Ruoff KL (1988) Streptococcus anginosus ("Streptococcus milleri"): the unrecognized pathogen. Clin Microbiol Rev 1: 102-108.

4. Bisno AL, Brito MO, Collins CM (2003) Molecular basis of group A Streptococcal virulence. Lancet Infect Dis 3: 191200.

5. Fischetti VA (1989) Streptococcal M Protein: Molecular Design and Biological Behavior. Clin Microbiol Rev 2: $285-$ 315.

6. Shet A, Kaplan E (2004) Addressing the burden of Group A Streptococcal diseases in India. Ind J Med Pediatr 71: 41-48.

7. Collee JG, Diguid JP, Fraser AG, editors (1996) Mackie and McCartney practical Medical Microbiology, 14thedition. Edinburgh: Churchill Livingstone. 263-273.

8. Clinical and Laboratory Standards Institute (2006) Performance standards for antimicrobial susceptibility testing. 16 suppl. M100-S16. CLSI: Wayne, PA.

9. Clinical Laboratory Standards Institute (2009) Performance standards for antimicrobial susceptibility testing. 19 Suppl. M100-S17. CLISI: Wayne, PA.

10. Jing HB, Ning B, Hao H, Zheng YL, Chang D, Jiang W, Jiang YQ (2006) Epidemiological analysis of group A streptococci recovered from patients in China. J Med Microbiol 55: 1101-1107.

11. Rivera A, Rebollo M, Miro E, Mateo M, Navarro F, Gurgui M, Mirelis B, Coll P (2006) Superantigen gene profile, emm type and antibiotic resistance genes among group A streptococcal isolates from Barcelona, Spain. J Med Microbiol 55: 1115-1123.

12. Beall B, Facklam R and Thompson T (1996) Sequencing emm-Specific PCR Products for Routine and Accurate Typing of Group A Streptococci. J Clin Microbiol 34: 953-958.

13. Mathur P, Kapil A, Das B (2004) Prevalence of group G \& group $\mathrm{C}$ streptococci at an Indian tertiary care centre. Indian J Med Res 120: 199-200.

14. Reissmann S, Friedrichs C, Rajkumari R, Itezek A, Fulde M, Rodloff AC, Brahmadathan KN, Chhatwal GS, NitscheSchmitz DP (2010) Contribution of Streptococcus anginosus to infections caused by groups $\mathrm{C}$ and $\mathrm{G}$ streptococci, southern India. Emerg Infect Dis 16: 656-663.

15. Bassetti M, Manno G, Collidà A, Ferrando A, Gatti G, Ugolotti, E, Cruciani M, Bassetti D (2000) Erythromycin resistance in Streptococcus pyogenes in Italy. Emerg Infect Dis 6: $180-183$.

16. York MY, Gibbs L, Perdreau-Remington F, Brooks GF (1999) Characterization of antimicrobial resistance in Streptococcus pyogenes isolates from the San Francisco bay area of northern California. J Clin Microbiol 37: 1727-1731.

17. Brown DF, Hope R, Livermore DM, Brick G, Broughton K, George RC, Reynolds R (2008) Non-susceptibility trends among enterococci and non-pneumococcal streptococci from bacteraemias in the UK and Ireland, 2001-06. J Antimicrob Chemother 62 Suppl. 2: 75-85.

18. Kittang BR, Skrede S, Langeland N, Haanshuus CG, Mylvaganam H (2011) Emm gene diversity, superantigen gene profiles and presence of SlaA among clinical isolates of group A, C and G streptococci from western Norway. Eur J Clin Microbiol Infect Dis 30: 423-433.

19. Hu MC, Walls MA, Stroop SD Reddish MA, Beall B, Dale JB (2002) Immunogenicity of a 26-valent group A Streptococcal vaccine. Infect Immun 70: 2171-2177.

20. Pimtanothai N, Nilgate S, Nunthapisud P (2002) Emm types of Invasive Group A Streptococcal Isolates from Thai Patients at King Chulalongkorn Memorial Hospital from 1995-1999. J Med Assoc Thai 85 Suppl 1: 371-377.

21. Sagar V, Bakshi D K, Nandi S, Ganguly NK, Kumar R, Chakraborti A (2004) Molecular heterogeneity among north Indian isolates of Group A Streptococcus. Lett Appl Microbiol 39: 84-88.

22. Smeesters PR, McMillan DJ, Sriprakash KS, Georgousakis MM (2009) Difference among group A Streptococcus epidemiological landscapes: consequences for $\mathrm{M}$ proteinbased vaccine? Expert Rev Vaccines 8: 1705-1720.

\section{Corresponding author}

Dr Purva Mathur,

Department of Laboratory Medicine, Jai Prakash Narayan Apex

Trauma Centre, All India Institute of Medical Sciences

New Delhi, 110029, India

Phone: 26189000, 26188000, and 26109000, Extn: 1169

Fax: 91-11-26106826

Email: purvamathur@yahoo.co.in

Conflict of interests: No conflict of interests is declared. 